\title{
Time-domain Monte Carlo simulation of GaN planar Gunn nanodiodes in resonant circuits
}

\author{
B. G. Vasallo , J. F. Millithaler, I. Íñiguez-de-la-Torre, T. González and J. Mateos \\ Dpto. Física Aplicada, Universidad de Salamanca, Spain \\ e-mail: bgvasallo@usal.es
}

\begin{abstract}
In this work we present a theoretical study based on time-domain Monte Carlo (MC) simulations of GaN-based Self-Switching Diodes (SSDs) oriented to the experimental achievement and control of the sub-THz Gunn-oscillations potentially provided by these devices. With this aim, an analysis of the frequency performance of SSDs connected to a resonant RLC parallel circuit, is reported here. V-shaped SSDs have been found to be more efficient, in terms of the DC to AC conversion efficiency $\eta$, than similar square-shape ones. Indeed, a value of $\eta$ of at least $0.80 \%$, can be achieved with appropriate RLC elements, even when considering heating effects. When the influence of parasitic elements such as the crosstalk capacitance $\mathrm{C}_{\text {talk }}$ is evaluated, MC simulations have shown that the resonant circuit must contain a capacitance $C$ higher than $C_{\text {talk }}$ in order to obtain experimentally useful values of $\eta$. This condition can be reached by integrating a sufficiently high number $N$ of parallel SSDs in the fabricated devices. MC simulations have also shown that when several diodes are fabricated in parallel the oscillations of all the SSDs are not synchronized, but this problem is solved by the attachment of a resonant RLC tank.
\end{abstract}

Keywords-Monte Carlo model, GaN Self-Switching Diodes, Gunn oscillations, resonant circuits.

\section{INTRODUCTION}

Planar Self-Switching Diodes (SSDs) [1] are being considered for $\mathrm{THz}$ signal emission at room temperature, since their topology is especially appropriate for the generation of Gunn oscillations [2]. Power emission can be improved by means of the fabrication of arrays of parallel channels, while enhanced broadband performance and flexibility of design can be achieved because of the possibility of fabricating together several devices with different characteristic frequencies. Moreover, the excellent transport properties of GaN allow envisaging the generation of Gunn oscillations approaching the $\mathrm{THz}$ range [3]. However, no experimental demonstration of this effect has been achieved so far (neither in vertical nor in planar diodes). In order to accomplish such experimental demonstration it seems necessary that Gunn diodes are embedded into a resonant circuit, which can be a simple parallel RLC circuit connected in series with the devices.

The main purpose of this work is to provide a Monte Carlo (MC) theoretical study of GaN SSDs connected in series with

This work has been partially supported by the Dirección General de Investigación Científica y Técnica (MINECO) through Project TEC201341640-R and by the Junta de Castilla y León through Project SA052U13. 978-1-4799-5433-9/14/\$31.00 (C2014 IEEE such a resonant circuit with the aim of identifying the optimum operation conditions for the experimental achievement of oscillations. Two kinds of geometries of GaN SSDs (the simple square and the optimized V-shaped) have been analyzed and compared in terms of oscillation frequencies and DC to $\mathrm{AC}$ conversion efficiency. As well, the advantages of parallelization have been explored, especially to overcome the negative effect of the parasitic crosstalk capacitance $\mathrm{C}_{\text {talk }}$.

\section{PHYSICAL MODEL}

For the calculations, we make use of a semiclassical ensemble MC simulator self-consistently coupled with a 2D Poisson solver whose validity for the analysis of the physical behavior of SSDs has already been demonstrated [3], [4]. Electron transport in $\mathrm{GaN}$ is modeled by three non-parabolic spherical valleys $\left(\Gamma_{1}, U\right.$ and $\left.\Gamma_{3}\right)$ with intervalley, acoustic and optical phonons, ionized impurities and piezoelectric scatterings [5]. The schematic top-view topologies of (a) the square (SQ-SSD) and (b) the V-shaped (VS-SSD) GaN diodes under analysis have been plotted in Fig. 1. To model the geometry of SSDs based on the $\mathrm{AlGaN} / \mathrm{GaN}$ heterojunction, the so-called top view simulations [4], [6]-[10] have been employed. This type of simulations includes the following two ad-hoc parameters: (i) a virtual background net doping $N_{D b}$ is assigned to the simulated $\mathrm{GaN}$ layer while the associated scattering is not considered, in order to account for the fixed charges at the top surface and the heterojunction. (ii) A negative surface charge density $\sigma$ is assigned to the semiconductor-air interfaces to account for the influence of the surface states originated during the etching process; this charge is updated consistently with the surrounding electron concentration in order to reproduce the correct behavior of the device [8]. A value of $N_{D b}=5 \times 10^{17} \mathrm{~cm}^{-3}$ has been used, while the non-simulated dimension employed to calculate the absolute values of current [4] has been considered to be $\mathrm{Z}=n_{s} / N_{D b}=0.1 \mu \mathrm{m}$ (taking as a base the experimental value of the sheet electron concentration, $n_{s}=5 \times 10^{12} \mathrm{~cm}^{-2}$ ).

In order to account for thermal effects, a self-consistent method making use of the thermal resistance of the structure has been implemented, the global lattice temperature $T_{\text {latt }}$ being iteratively adapted self-consistently with the power dissipated within the device [11]. A value of $R_{t h}=10 \times 10^{-4} \mathrm{~K} /(\mathrm{W} / \mathrm{m})$ has been found to well reproduce the experimental I-V curves of the real devices when thermal effects are significant. Under these conditions, the simulations show that the devices reach a maximum $T_{\text {latt }}$ of about $500 \mathrm{~K}$ at $20 \mathrm{~V}$ (also in good agreement with the experimental findings). MC simulations of Gunn 

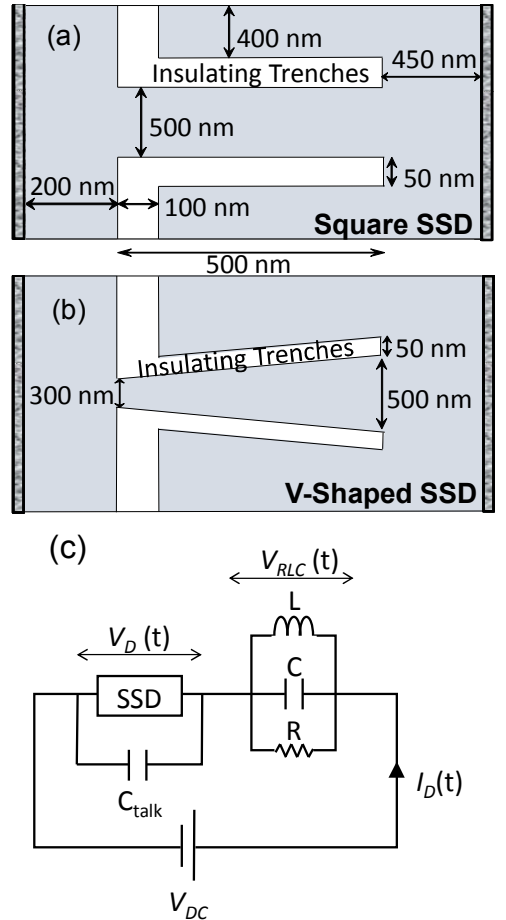

Fig. 1. Sketch of the topology of the GaN (a) SQ SSD and (b) VS SSD under analysis. (c) Circuit configuration of the series connection of the SSD with the parallel RLC tank.

oscillations in GaN SSDs including thermal effects show that the heating leads to a decrease of the oscillation frequency due to a lower drift velocity of the Gunn domain [11], [12].

The intrinsic MC simulation of the diode has been coupled to the resolution of a parallel RLC resonant circuit connected in series [13], as sketched in Fig. 1(c). The coupling of the circuit equations with the $\mathrm{MC}$ simulator is carried out in a discrete time mesh using the same time step as the simulator. The circuit equations to be solved are the following:

$$
V_{D C}=V_{D}+V_{R L C},
$$

where $V_{D}$ and $V_{R L C}$ are the voltage drops at the terminals of the diode and the RLC circuit, respectively. The total current flowing through the $\mathrm{SSD}, j_{D}$, is:

$$
j_{D}=\mathrm{C} d V_{R L C} /_{d t}+j_{L}+V_{R L C} /_{R},
$$

being $j_{L}$ the current flowing through the inductance L, which can be obtained through:

$$
V_{R L C}=\mathrm{L}^{d j_{L}} / d t
$$

The total current flowing through the diode has two components:

$$
j_{D}=j_{v}+\mathrm{C}_{\mathrm{D}} d V_{D} / d t
$$

the drift component of the current $j_{v}$ is directly calculated by the $\mathrm{MC}$ technique from the particle velocities following the generalized Ramo-Shockley theorem [14], and the displacement current $\mathrm{C}_{\mathrm{D}} d V_{D} / d t$ is due to the time-varying potentials at the electrodes of the diode, being $\mathrm{C}_{\mathrm{D}}=0.0057 \mathrm{fF}$ the diode intrinsic geometric capacitance. The parasitic effect of the crosstalk capacitance $\mathrm{C}_{\text {talk }}$ is accounted for by adding its value to $C_{D}$ [Fig. 1(c)]. The coupling of the circuit equations with the MC simulator is carried out following a finitedifference time-domain scheme, using the same time step as the simulator. The voltage drop at the terminals of the diode $V_{D}$, used to solve the Poisson equation, is updated at each time step with the solution of the previous system of equations obtained with the value of $j_{v}$ provided by the MC simulation [15].

The results of MC simulations in which one single diode is considered (embedded in resonant circuit with $\mathrm{R}_{1}, \mathrm{~L}_{1}$ and $\mathrm{C}_{1}$ ) can be extrapolated to the case of the parallelization of N SSDs (normally designed to decrease their high impedance and increase the delivered power), as long as a resonant circuit with $\mathrm{R}_{\mathrm{N}}=\mathrm{R}_{1} / \mathrm{N}, \mathrm{L}_{\mathrm{N}}=\mathrm{L}_{1} / \mathrm{N}$ and $\mathrm{C}_{\mathrm{N}}=\mathrm{C}_{1} \times \mathrm{N}$ is used.

Finally, in order to analyze the diodes capability as emitters, the dissipated DC power $\left(P_{D C}\right)$ and the time-average AC power $\left(P_{A C}\right)$ are evaluated, and the conversion efficiency is calculated as $\eta=-P_{A C} / P_{D C}$ (positive values of $\eta$ indicating $\mathrm{AC}$ generation from $\mathrm{DC}$, while negative values indicate a resistive behavior of the diode).

\section{RESULTS}

\section{A. Efficiency and frequency of oscillations}

Fig. 2 presents the efficiency $\eta$ and the frequency of the current oscillations directly obtained by $\mathrm{MC}$ simulations when an RLC parallel circuit with $\mathrm{R}=0.5 \mathrm{M} \Omega, \mathrm{C}=0.01 \mathrm{fF}$ and $\mathrm{L}$ varying from 5 to $100 \mathrm{nH}$ has been modeled in series with a single SSD. The negative differential resistance of the SSD (also calculated by means of MC simulations, following the method in [16]) combined with the feedback of the resonant circuit, leads to oscillations at a frequency lower than the characteristic resonant frequency of the RLC circuit and, as well, lower than that of Gunn self-oscillations (around $385 \mathrm{GHz}$ ). To further illustrate this, Fig. 3 presents the time evolution of the current through the diode (a) without and (b) embedded in the mentioned resonat circuit for $V_{D C}=30 \mathrm{~V}$, being $\mathrm{R}=0.5 \mathrm{M} \Omega, \mathrm{C}=0.01 \mathrm{fF}$ and $\mathrm{L}=12 \mathrm{nH}$, for which a maximum efficiency of $0.18 \%$ has been found (providing oscillations at $275 \mathrm{GHz}$ ). However, we have to stress that the time consuming MC simulations only allowed us to check a limited set of combinations of $\mathrm{R}, \mathrm{L}$ and $\mathrm{C}$ values. The acshieved conversion efficiencies are consistent with other theoretical predictions in submicrometer GaN Gunn diodes [17]. In addition, a large frequency tunability (from 270 to $350 \mathrm{GHz}$ ) can be achieved at the expense of lower efficiencies. These low values of $\eta$, even if they could be already experimentally useful, can be further enhanced by optimizing the values of $\mathrm{R}, \mathrm{L}$ and $\mathrm{C}$, by using different circuit configurations, and as well by improving the intrinsic characteristics of the SSD. 


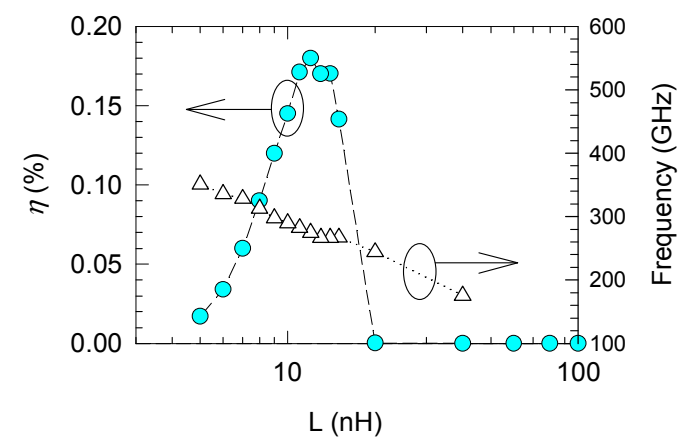

Fig. 2. DC to AC conversion efficiency, $\eta$, and frequency of the current oscillations obtained with MC simulations in the SQ-SSD sketched in Fig. 1(a) connected in series with a resonant circuit with $\mathrm{R}=0.5 \mathrm{M} \Omega$, $\mathrm{C}=0.01 \mathrm{fF}$ and $\mathrm{L}$ varying from 5 to $100 \mathrm{nH}$, at $300 \mathrm{~K} . V_{D C}=30 \mathrm{~V}$.

In order to establish a comparison between the SQ- and VSSSDs, the dynamic behavior of both SSDs coupled with the same resonant circuit with $\mathrm{R}=0.5 \mathrm{M} \Omega, \mathrm{C}=0.01 \mathrm{fF}$ and $\mathrm{L}=12 \mathrm{nH}$ has been analyzed for different DC biasing conditions (Fig. 4). At $300 \mathrm{~K}$ the VS-SSD exhibits a threshold voltage for the onset of oscillations of $V_{D C}=20 \mathrm{~V}$, lower than in the SQ-SSD, which needs $V_{D C}>25 \mathrm{~V}$. The oscillation frequencies are in the range 265-275 GHz in the VS-SSD, just slightly dependent on $\mathrm{V}_{\mathrm{DC}}$ and with lower values than for the SQ-SSD (which provides 275-290 GHz). For both kinds of diodes, the efficiency presents a maximum value at $V_{D C}=30 \mathrm{~V}$, which is significantly enhanced for the VS-SSD (around 0.35\%). Surprisingly, the efficiency obtained when heating effects are considered is higher than at $300 \mathrm{~K}$, reaching a maximum of around $0.6 \%$ for the VS-SSD (at a $T_{\text {latt }}$ higher than $450 \mathrm{~K}$ ) with similar oscillation frequencies, which are practically fixed by the external circuit. This can be attributed to the fact that the particular values of $\mathrm{R}, \mathrm{L}$ and $\mathrm{C}$ used for these $\mathrm{MC}$ simulations are not optimized for the devices at $300 \mathrm{~K}$, but they better match the characteristics of the SSDs when operating at high temperature. Indeed, as shown in Fig. 5, the efficiency of the VS-SSD can be increased to $0.72 \%$ at $V_{D C}=25 \mathrm{~V}$ with $\mathrm{C}=0.022 \mathrm{fF}$ and $\mathrm{L}=12 \mathrm{nH}$ (even to $0.8 \%$ by increasing both $\mathrm{C}$ and $\mathrm{L}$ to $\mathrm{C}=0.014 \mathrm{fF}$ and $\mathrm{L}=16 \mathrm{nH}$ ), using a higher capacitance that may help to compensate the inhibiting oscillation effect of the crosstalk (that will be discussed later), since for a fabricated SQ-SSD array of $\mathrm{N}=25$ the value of $\mathrm{C}_{\text {talk }}$ is already $5.5 \mathrm{fF}$.

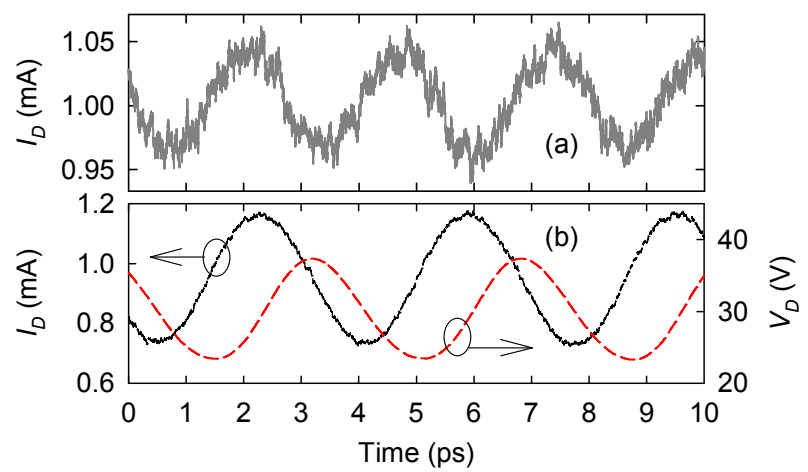

Fig. 3. Time dependence of (a) $I_{D}$, and (b) $I_{D}$ and $V_{D}$ in the SQ-SSD sketched in Fig. 1(a), (a) in the absence and (b) connected in series with a resonant circuit with $\mathrm{R}=0.5 \mathrm{M} \Omega, \mathrm{C}=0.01 \mathrm{fF}$ and $\mathrm{L}=12 \mathrm{nH}$, at $300 \mathrm{~K}$. $V_{D C}=30 \mathrm{~V}$.

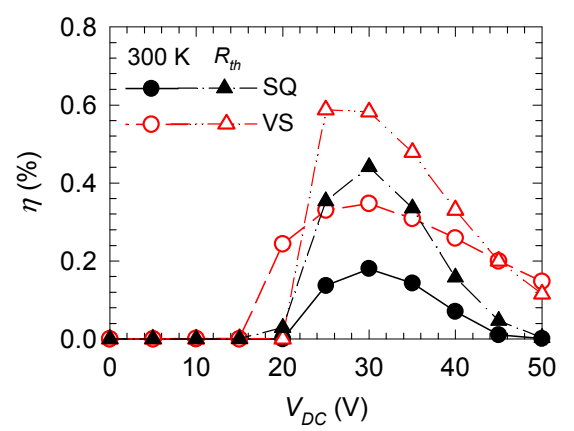

Fig. 4. DC to AC conversion efficiency, $\eta$, as a function of the applied DC voltage obtained with the MC simulations of the SQ- and VS-SSD sketched in Fig. 1, connected in series with a RLC tank with $\mathrm{R}=0.5 \mathrm{M} \Omega, \mathrm{C}=0.01 \mathrm{fF}$ and $\mathrm{L}=12 \mathrm{nH}$. The results at $300 \mathrm{~K}$ are compared with those obtained with the self-consistent thermal MC model considering $\mathrm{R}_{\mathrm{th}}=10 \times 10^{4} \mathrm{~K} /(\mathrm{W} / \mathrm{m})$.

Another interesting result of the employment of an RLC resonant circuit in series with $\mathrm{N}$ parallel SSDs is that it ensures the coherence between the Gunn oscillations of the individual channels, avoiding the desynchronization that appears when just a DC bias is applied to the array. As a consequence, the $\mathrm{DC}$ to $\mathrm{AC}$ conversion efficiency is independent of $\mathrm{N}$ as long as the values of the elements of the resonant circuit are scaled as: $\mathrm{R}_{\mathrm{N}}=\mathrm{R}_{1} / \mathrm{N}, \mathrm{L}_{\mathrm{N}}=\mathrm{L}_{1} / \mathrm{N}$ and $\mathrm{C}_{\mathrm{N}}=\mathrm{C}_{1} \times \mathrm{N}$.
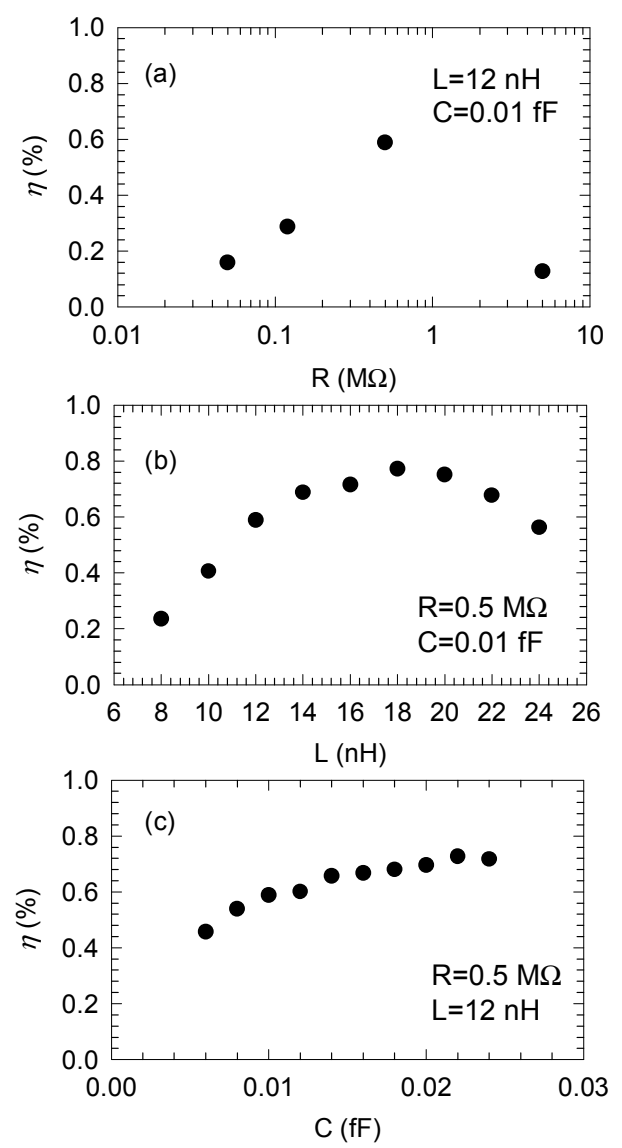

Fig. 5. DC to AC conversion efficiency, $\eta$, for the VS-SSD sketched in Fig. 1(b) connected in series with a resonant circuit with (a) $C=0.01 \mathrm{fF}, \mathrm{L}=12 \mathrm{nH}$ and several values of $\mathrm{R}$, (b) $\mathrm{R}=0.5 \mathrm{M} \Omega, \mathrm{C}=0.01 \mathrm{fF}$ and several values of $\mathrm{L}$, and (b) $\mathrm{R}=0.5 \mathrm{M} \Omega, \mathrm{L}=1.2 \mathrm{nH}$ and several values of $\mathrm{C}$, obtained with the selfconsistent thermal MC model considering $\mathrm{R}_{\mathrm{th}}=10 \times 10^{4} \mathrm{~K} /(\mathrm{W} / \mathrm{m}) . V_{D C}=25 \mathrm{~V}$. 


\section{B. Effect of the crosstalk capacitance, $\mathrm{C}_{\mathrm{talk}}$}

The effect of different values of $\mathrm{C}_{\text {talk }}$ has been evaluated for the SQ- and VS-SSDs at room temperature embedded in a resonant circuit with $\mathrm{R}=0.5 \mathrm{M} \Omega, \mathrm{C}=0.01 \mathrm{fF}$ and $\mathrm{L}=12 \mathrm{nH}$, for $V_{D S}=30 \mathrm{~V}$. In Fig. 6, $\eta$ as a function of $\mathrm{C}_{\text {talk }}$ has been represented. For both devices, when the value of $\mathrm{C}_{\text {talk }}$ is higher than that of $\mathrm{C}$, the efficiency drops and the oscillations finally disappear. For the VS-SSD the decrease of $\eta$ when increasing $\mathrm{C}_{\text {talk }}$ is sharper, further showing a more robust performance than the SQ-SSD. In any case, the effect of $\mathrm{C}_{\text {talk }}$ is a severe problem for the experimental achievement of current oscillations, since the condition $\mathrm{C}<\mathrm{C}_{\text {talk }}$ is not easy to fulfill. In fact, $\mathrm{C}_{\text {talk }}$ has been experimentally estimated to be about $5 \mathrm{fF}$ (being almost independent of the number $\mathrm{N}$ of SSDs in parallel, since it is mainly determined by the geometry of the mesa and access regions) and it can be at the origin of the absence of experimental evidence of Gunn oscillations in the fabricated devices.

As a result, for the current technology oscillations can only be generated by using an RLC circuit with a value of $\mathrm{C}$ higher than $5 \mathrm{fF}$. This problem can be overcome by using arrays of $\mathrm{N}$ parallel SSD since the capacitance to be used is $\mathrm{C}_{\mathrm{N}}=\mathrm{C}_{1} \times \mathrm{N}$. However, in order to fulfill the condition $\mathrm{C}>\mathrm{C}_{\text {talk }}, \mathrm{N}$ has to be increased to $>500$, so that the thermal management may be problematic. Technologically, the crosstalk capacitances can be decreased by improving the design of the accesses and moving the ohmic contacts further away, so that the number of channels to have $\mathrm{C}>\mathrm{C}_{\text {talk }}$ can be reduced as much as possible. Furthermore, other circuit configurations of the resonator using higher capacitances could be used in order to reduce the influence of $\mathrm{C}_{\text {talk }}$.

\section{CONCLUSIONS}

A semiclassical ensemble MC simulator self-consistently coupled with a 2D Poisson solver has been employed to analyzed the Gunn-oscillation operation of GaN-based SQ- and VS-SSDs working in series with a resonant RLC parallel circuit in terms of the DC to AC conversion efficiency and the current-oscillation frequency. With SQ-SSDs an efficiency of at least $0.18 \%$ can be achieved, with the possibility of frequency tunability from 270 to $350 \mathrm{GHz}$, at $300 \mathrm{~K}$. VS-SSDs have been found to be more efficient than SQ-SSDs, providing a value of $\eta$ of at least $0.35 \%$. This value can be increased up to at least $0.80 \%$, even when considering the degradation of the oscillating behavior produced by self-heating effects. The

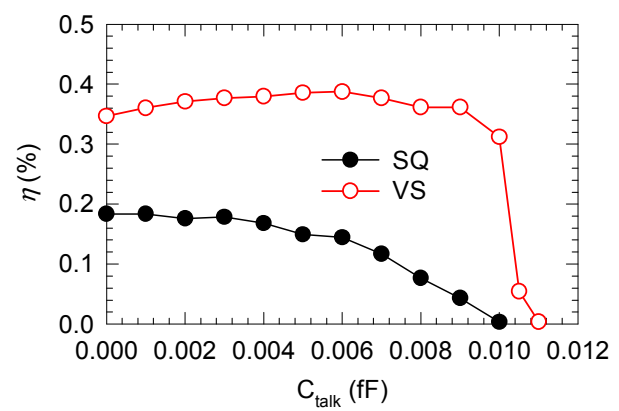

Fig. 6. DC to AC conversion efficiency, $\eta$, at $300 \mathrm{~K}$ in the SSDs of Fig. 1 connected in series with a resonant circuit with $\mathrm{R}=0.5 \mathrm{M} \Omega, \mathrm{C}=0.01 \mathrm{fF}$ and $\mathrm{L}=1.2 \mathrm{nH}$ at room temperature. $V_{D C}=30 \mathrm{~V}$. negative effect of the crosstalk capacitance may be overcome by using an array of $\mathrm{N}$ parallel SSDs, that allows the use of $\mathrm{C}>\mathrm{C}_{\text {talk }}$ in the resonant circuit.

\section{REFERENCES}

[1] Terahertz Frequency Detection and Identification of Materials and Objects, edited by R. E. Miles, X. -C. Zhang, H. Eisele, and A. Krotkus, New York: Springer, 2007.

[2] M. Song, M. Missous, P. Omling, A. R. Peaker, L. Samuelson, and W. Seifert, "Unidirectional electron flow in a nanometer-scale semiconductor channel: A self-switching device," Appl. Phys. Lett., vol. 83, pp. 1881-1883, Sept. 2003.

[3] K. Y. Xu, G. Wang and A. M. Song, "Gunn oscillations in a selfswitching nanodiode," Appl. Phys. Lett., vol. 93, 233506 (3 pp.), Dec. 2008.

[4] J. Mateos, B. G. Vasallo, D. Pardo and T. González, "Operation of highfrequency performance of nanoscale unipolar rectifying diode," Appl. Phys. Lett., vol. 86, 212103 (3 pp.), May 2005.

[5] S. García, S. Pérez, I. Iñiguez-de-la-Torre, J. Mateos, and T. González, "Comparative Monte Carlo analysis on InP- and GaN-based Gunn diodes,” J. Appl. Phys., vol. 115, 044510 (7 pp.), Jan. 2014.

[6] J. Mateos, B. G. Vasallo, D. Pardo, T. González, J. S. Galloo., Y. Roelens, S. Bollaert and A. Cappy, "Ballistic nanodevices for terahertz data processing: Monte Carlo simulations," Nanotechnology, vol. 14, pp. 117-122, Jan. 2003.

[7] J. Mateos, B. G. Vasallo, D. Pardo, T. González, J. S. Galloo, S. Bollaert, Y. Roelens and A. Cappy, "Microscopic Modeling of Nonlinear Transport in Ballistic Nanodevices," IEEE Trans. Electron Devices, vol. 50, pp. 1897-1905, Sep. 2003.

[8] I. Iñiguez-de-la-Torre, J. Mateos, T. González, D. Pardo, J. S. Galloo, S. Bollaert, Y. Roelens and A. Cappy, "Influence of the surface charge on the operation of ballistic T-branch junctions: a self-consistent model for Monte Carlo simulations," Semicond. Sci. Technol., vol. 22, pp. 663, June 2007.

[9] K. Y. Xu, X. F. Lu., A. M. Song and G. Wang, "Terahertz harmonic generation using planar nanoscale unipolar diode at zero bias," Appl. Phys. Lett., vol. 92, 163503 (3 pp.), Apr. 2008.

[10] A. Iñiguez-de-la-Torre, I. Iñiguez-de-la-Torre, J. Mateos, T. González, P. Sangaré, M. Gaucher, B. Grimbert, V. Brandli, G. Ducournau and C. Gaquière, "Searching for $\mathrm{THz}$ Gunn oscillations in $\mathrm{GaN}$ planar nanodiodes," J. Appl. Phys., vol. 111, 113705 (9 pp.), June 2012.

[11] J. F. Millithaler, I. Íñiguez-de-la-Torre, T. González, J. Mateos, P. Sangaré. G. Ducournau, C. Gaquière, "Monte Carlo analysis of thermal effects in Self-switching diodes," Spanish Conference on Electron Devices, pp. 45-48, 2013. DOI: 10.1109/CDE.2013.6481338.

[12] J.-F. Millithaler, I. Iñiguez-de-la-Torre, A. Iñiguez-de-la-Torre, T. González, P. Sangaré, G. Ducournau, C. Gaquière and J. Mateos, "Optimized V-shape design of GaN nanodiodes for the generation of Gunn oscillations,” Appl. Phys. Lett., vol. 104, 073509 (3 pp.), Feb. 2014.

[13] P. Shiktorov, E. Starikov, V. Gruzinskis, S. Pérez, T. González, L. Reggiani, L. Varani and J. C. Vaissière, "Theoretical investigation of Schottky-barrier diode noise performance in external resonant circuits," Semicond. Sci. Technol., vol. 21, pp. 550-557, April 2006.

[14] H. Kim, H. S. Min, T. W. Tang and Y. J. Park, "An extended proof of the Ramo-Shockley theorem," Solid-State Electron., vol. 34, pp. 12511253, May 1991.

[15] S. García, B. G. Vasallo, J. Mateos and T. González, "Time-domain Monte Carlo simulations of resonant-circuit operation of GaN Gunn diodes," Spanish Conference on Electron Devices, pp. 79-82, 2013. DOI: 10.1109/CDE.2013.6481347.

[16] G.M. Dunn, A. Phillips and P. J. Topham, "Current instability in power HEMTs," Semicond. Sci. Technol., vol. 16, pp. 562-566, Apr. 2001.

[17] E. A. Barry, V. N. Sokolov, K. W. Kim and R. J. Trew, "Large-signal analysis of terahertz generation in submicrometer GaN diodes," IEEE Sensor Journal, vol. 10, pp. 765-771, March 2010. 